\title{
Operational Characteristics of a GEM- MSGC System for X-ray Detection
}

\section{J.A. Mir}

15 July 2004 


\section{(c) Council for the Central Laboratory of the Research Councils}

Enquiries about copyright, reproduction and requests for additional copies of this report should be addressed to:

Library and Information Services

CCLRC Rutherford Appleton Laboratory

Chilton Didcot

Oxfordshire OX1100X

UK

Tel: $+44(0) 1235445384$

Fax: $+44(0) 1235446403$

Email: library@rl.ac.uk

CCLRC reports are avallable online at:

http:/WWW. clrc.ac.uk/Activity/ACTIVTY=Publications; SECTION=225;

ISSN 1358-6254

Neither the Council nor the Laboratory accept any responsibility for loss or damage arising from the use of information contained in any of their reports or in any communication about their tests or investigations. 
RAL-TR-2004-019

\title{
Operational Characteristics of a GEM-MSGC System for X-ray Detection
}

\author{
J.A. $\operatorname{Mir}^{*}$ \\ CCLRC Rutherford Appleton Laboratory, Chilton, Didcot, Oxon, OX11 0QX, UK \\ $11^{\text {th }}$ May 2004
}

\begin{abstract}
:
This report outlines a recent study undertaken at CCLRC Rutherford Appleton Laboratory to evaluate the performance of a Gas Electron Multiplier (GEM) coupled with a Microstrip Gas counter (MSGC). The parameters investigated during this study were effective gain, effective gain stability and energy resolution at $8.05 \mathrm{keV}$ using $\mathrm{Cu}-\mathrm{K} \mathrm{X}$-rays. These parameters were studied as a function of drift field, induction field, potential differences across the GEM holes and that across the MSGC anodes and cathodes. This report demonstrates that a single stage GEM can sustain effective gains up to 6000 whilst retaining adequate X-ray energy resolution. By utilising the MSGC as well as the GEM amplification these gains easily exceed 100,000 and allow the MSGC operation at much lower voltages. This report also demonstrates that the introduction of the GEM preamplification to the MSGC enables the operation of the latter at much higher effective gains $(30,000)$ before any degradation in the X-ray energy resolution.
\end{abstract}

*Corresponding Author: Tel : 44-(0)-1235-44-6262, Email: J.A.Mir@rl.ac.uk 


\subsection{Introduction}

The gas electron multiplier (GEM) was invented at CERN by F. Sauli in 1997 [1] and consists of a thin polymer mesh ( 50 micron thick kapton), metal clad on both sides usually by 5 micron thick copper and perforated with a high density of holes (Typically 40-120 micron hole diameter, 140-200 micron hole pitch). The GEM mesh fabrication involves conventional phototlihography using a double mask process where hole patterns are engraved on both sides of the copper clad kapton. The GEM channels are then opened up using a kapton specific etching agent that dissolves the unprotected kapton areas leaving double conical shaped GEM holes. Upon the application of a potential difference across the GEM holes $\left(\Delta_{\mathrm{GEM}}\right)$ in a suitable counter gas, charge amplification is achieved when electrons produced from the radiation above the GEM plane enter the holes. Over the past few years the GEM technology has become widespread and found applications in a wide range of fields such as high energy physics experiments such as COMPASS [2], LEGS TPC [3], ultra-fast soft X-ray plasma diagnostics [4] and neutron detection [5 and 6].

In this study, a GEM-MSGC system was used to examine the relative pulse heights at the MSGC anodes as a function of drift field, $\mathrm{E}_{\mathrm{d}}$, induction field, $\mathrm{E}$ and the voltage applied across the GEM holes, $\Delta_{\text {GEM}}$. Other parameters studied using the present system were the effective gain and the X-ray energy resolution as a function of the induction field, the voltage applied across the GEM holes and the voltage applied at the MSGC cathodes, $V_{c}$ or anodes, $V_{a}$. The short term effective gain stability of the GEM-MSGC system was also explored as a function of the counter gas mixture and the induction field.

\subsection{Method}

The experimental arrangement used during the present study is shown in figure 1. It consisted of a MSGC plate described in a previous experimental investigation [7], a GEM mesh and a drift plate. The MSGC section used throughout this work consisted of 5 microns wide anodes, 200 microns wide cathodes set at 500 microns pitch. The active area of the MSGC was approximately $6 \mathrm{~mm}$ by $18 \mathrm{~mm}$. The GEM mesh in this study consisted of 70 (55) micron holes at 140 micron pitch (see figure 1) using a 50 micron thick kapton foil. The separation between the GEM plane and the MSGC plate was held at $6 \mathrm{~mm}$ (Induction region) whereas the distance between the GEM plane and the drift plane was also held at $6 \mathrm{~mm}$ (Drift /Conversion region).

In the tests described here, a Cu-K X-ray $(8.05 \mathrm{keV})$ beam illuminated the detector drift space parallel to the GEM plane through a mylar window at the end wall of the detector chamber. The mesh window consisted of $100 \mu \mathrm{m}$ mylar mesh with $100 \mathrm{~nm}$ aluminium coating on one side. The X-ray beam passed over the GEM plane and allowed the investigations of the effective gain and the energy resolution for a variety of experimental conditions. An argon (75\%) and Isobutane (IB) (25\%) counter gas mixture was used through most of this study with the exception of one test where $\operatorname{Ar}(52 \%)$-Dimethylether (DME) (48\%) was used. The gas mixture was controlled by Brooks mass-flow controllers (model $5850 \mathrm{E}$ ) built into a rig fabricated in stainless steel tubing which is used throughout with the exception of small sections of flexible nylon tubing (Type NT, BS5409) close to the detector to facilitate mounting. The absolute accuracy of the gas mixing is given as $1 \%$ with a repeatability of $0.25 \%$ of the flow. The ambient pressure and the temperature of the detector box were recorded with every measurement.

The initial X-ray interaction between the X-ray beam and counter gas atoms produces a primary cluster containing $\mathrm{N}_{\mathrm{o}}$ electrons per event. This number is governed by the Poisson 
In this part of the study the relative pulse height and the X-ray energy resolution, at $8.05 \mathrm{keV}$, at the MSGC anodes were examined as a function of the drift field for a range of voltages across the GEM holes, $\Delta_{\mathrm{GEM}}$, and induction fields, $\mathrm{E}_{\mathrm{I}}$. Figure 2 shows typical trends of these parameters with increasing drift fields with $\Delta_{\mathrm{GEM}}=400 \mathrm{~V}: \mathrm{E}_{\mathrm{I}}=2.5 \mathrm{kV} / \mathrm{cm}$. As can be seen from figure 2, the relative pulse height drops significantly at the lowest and the highest drift fields accompanied by deterioration in the X-ray energy resolution. At the lowest drift fields, the pulse height reductions are attributed to electron losses due to attachment to electronegative impurities as well as losses due to electron diffusion to the GEM channel walls. At the highest fields some drift field lines begin to terminate at the topside of the GEM mesh causing electrons to impinge there resulting in the observed pulse height reductions.

Figure 3 shows the variation of the relative pulse height at the MSGC anodes as a function of drift field for $\Delta_{\mathrm{GEM}}=250 \mathrm{~V}: \mathrm{E}=2.5 \mathrm{kV} / \mathrm{cm}, \Delta_{\mathrm{GEM}}=250 \mathrm{~V}: \mathrm{E}_{\mathrm{I}}=4.2 \mathrm{kV} / \mathrm{cm}, \Delta_{\mathrm{GEM}}=400 \mathrm{~V}: \mathrm{E}_{\mathrm{I}}=$ $2.5 \mathrm{kV} / \mathrm{cm}$ and $\Delta_{\mathrm{GEM}}=500 \mathrm{~V}: \mathrm{E}=2.5 \mathrm{kV} / \mathrm{cm}$. Figure 3 demonstrates that the region of good electron transparency, i.e. the plateau, is unaffected by altering the induction field. In contrast, the region of good electron transparency is extended by applying higher voltages across the GEM holes.

It is worth noting that the analogous nature of the electrical transparency with optical transparency. The optical transparency $\tau$ of a GEM mesh is defined by the ratio of open to total area and can be expressed as $\tau=\pi D^{2} / 2(\sqrt{3})(P)^{2}$ where $D$ is the GEM hole diameter with hole pitch $\mathrm{P}$. Thus for the present GEM mesh $\tau=0.23$ as $\mathrm{D}=70$ microns and $\mathrm{P}=140$ microns. Increasing optical transparency by either increasing GEM hole diameter or decreasing GEM hole pitch also extends the useful drift field region (plateau) of figures 2 and 3 . These design changes enable GEM operation at even higher drift fields without suffering electron loss at the topside of the GEM mesh. These simple measurements provide important information about, for instance, the geometry needed to operate the GEM in a high magnetic field environment where the use of high drift is compulsory to reduce the Lorentz angle. In this respect, others [9] have used coarser GEM holes ( $\tau=0.38, \mathrm{D}=90$ microns and $\mathrm{P}=140$ microns $)$ to accomplish GEM operation at drift fields approaching $5 \mathrm{kV} / \mathrm{cm}$.

\subsection{Effective Gain and Energy Resolution Studies}

Induction Field: For a fixed drift field, electrons amplified in GEM holes are shared between the bottom side of the GEM mesh $\left(\mathrm{Q}_{\mathrm{b}}\right)$ and the MSGC anodes $\left(\mathrm{Q}_{\mathrm{a}}\right)$. In this study, the dependence of effective gain $\left(\mathrm{Q}_{\mathrm{a}} / \mathrm{N}_{\mathrm{o}} \mathrm{q}\right)$ upon the induction field was examined for $\Delta_{\mathrm{GEM}} 250 \mathrm{~V}$ and $350 \mathrm{~V}$ while keeping drift field $\mathrm{E}_{\mathrm{d}}$ and the MSGC cathode voltage $\mathrm{V}_{\mathrm{c}}$ constant at approximately $-0.25 \mathrm{kV} / \mathrm{cm}$ and $-622 \mathrm{~V}$, respectively. Figure 4 shows the variation of the effective gain and the $\mathrm{X}$-ray energy resolution at $8.05 \mathrm{keV}$ as a function of the induction field for the range $\mathrm{E}=1 \mathrm{kV} / \mathrm{cm}$ to $5 \mathrm{kV} / \mathrm{cm}$. This figure illustrates the importance of optimising the induction field of the GEM by the virtue of the increased effective gains with increasing induction fields.

The effective gain increases almost linearly with induction field for the induction field range explored here. This linear relationship however does not hold indefinitely as at higher induction field $\left(\mathrm{E}_{\mathrm{I}}>10 \mathrm{kV} / \mathrm{cm}\right)$ [9], the GEM mesh enters a parallel plate amplification mode with runaway and unstable gains. Figure 4 also shows that the $\mathrm{X}$-ray energy resolution improves when $\Delta_{\mathrm{GEM}}$ is increased from $250 \mathrm{~V}$ to $350 \mathrm{~V}$ and that an optimum X-ray resolution $(18 \% \mathrm{FWHM}$ at $8.05 \mathrm{keV})$ is obtained when induction field exceeds $2 \mathrm{kV} / \mathrm{cm}$. 
MSGC Voltage: Figure 5 shows the variation of the effective gain as a function of the MSGC anode voltage for a number of different voltages across the GEM holes $\left(\Delta_{\mathrm{GEM}}=250 \mathrm{~V}, 350 \mathrm{~V}\right.$ and $500 \mathrm{~V}$ ). The drift field during these measurements was maintained at $\sim-0.33 \mathrm{kV} / \mathrm{cm}$ whilst the induction field was kept at $\sim 2.5 \mathrm{kV} / \mathrm{cm}$. For making gain comparisons, figure 5 also shows the real gain of the MSGC without the deployment of the GEM mesh, denoted here by $\left(\Delta_{\mathrm{GEM}}=\right.$ $0 \mathrm{~V})$. Thus by using the present GEM-MSGC combination, the overall effective gain at $\Delta_{\mathrm{GEM}}=$ $500 \mathrm{~V}$ has increased by three orders of magnitude than that obtained by the sole MSGC operation. Another obvious consequence of utilising the additional charge amplification from the GEM mesh is the resulting shift of the gain-voltage characteristics of the MSGC to much lower voltages (figure 5).

The effective gain versus the MSGC anode voltage curves shown in figure 5 exhibit two different features. For example, the effective gain grows exponentially up to approximately a gain of 30,000 which represents a total charge in a single avalanche of $8.6 \times 10^{6}$ electrons $\left(30,000 * \mathrm{~N}_{0}=30,000 * 288\right)$ at the MSGC anodes. Thereafter, the effective gain increases almost linearly with increasing MSGC voltage surpassing values above 100,000 . This transition is indicative of the onset of space charge effects that generally lead to gain sag as well as deterioration in the $\mathrm{X}$-ray energy resolution.

The X-ray energy resolutions at $8.05 \mathrm{keV}$ associated with figure 5 are shown in figure 6 as a function of the effective gain. Previous studies using the MSGC alone [7 and 10] had showed that the X-ray energy resolution starts to degrade at gains exceeding approximately 2,000 culminating in severe distortions of the pulse height spectrum above gains of 20,000 due to the onset of space charge effects. Indeed, the X-energy resolution degradation at low gains (2000) using the MSGC alone in this study is also evident as shown in figure $6\left(\Delta_{\mathrm{GEM}}=0 \mathrm{~V}\right)$. The optimum X-ray energy resolution at $8.05 \mathrm{keV}$ was found to be approximately $16 \% \mathrm{FWHM}$ using the MSGC alone $\left(\Delta_{\mathrm{GEM}}=0 \mathrm{~V}\right)$ compared with the calculated value of $12 \% \mathrm{FWHM}$ at this energy. This discrepancy will be subject of future investigations.

By introducing a GEM preamplification stage, pulse height spectrum remains distortion-free and adequate X-ray energy resolutions are achieved up to gains of 30,000. This observation is consistent with earlier work on multistep multiwire proportional counters where these detectors were required to achieve highest spatial resolution whilst retaining a good X-ray energy resolution [10 and 11]. Presently, using the GEM-MSGC combination at an effective gain of 30,000 , the X-ray energy resolution was found to be approximately $18 \% \mathrm{FWHM}$ at $8.05 \mathrm{keV}$.

GEM Voltage: Figure 7 shows the variation of the effective gain as a function of the voltage applied across the GEM holes, $\Delta_{\mathrm{GEM}}$, for a number of different MSGC anode voltages $\left(\mathrm{V}_{\mathrm{a}}=\right.$ $+250 \mathrm{~V},+465 \mathrm{~V}$ and $+622 \mathrm{~V}$ ). The drift field during these measurements was maintained at approximately $-0.33 \mathrm{kV} / \mathrm{cm}$ whilst the induction field was at $2.5 \mathrm{kV} / \mathrm{cm}$. The curve corresponding to the MSGC anode voltage $\mathrm{V}_{\mathrm{a}}=+250 \mathrm{~V}$ represents zero gain contribution from the MSGC as shown in figure 5. The GEM mesh alone was therefore able to sustain an effective gain in excess of 6000 as shown in figure $7\left(\mathrm{~V}_{\mathrm{a}}=+250 \mathrm{~V}\right)$. Figure 7 also shows similar gain characteristics to those shown in figure 5 whereby effective gain saturation is observed beyond a value of 30,000 .

The associated X-ray energy resolution at $8.05 \mathrm{keV}$ for each curve in figure 7 is plotted in figure 8. Figure 9 show a typical height distribution using the $8.05 \mathrm{keV} \mathrm{Cu}-\mathrm{K} \mathrm{X}$-ray beam when the GEM mesh was operated at $\Delta_{\mathrm{GEM}}=500 \mathrm{~V}$ with the MSGC anodes at $+250 \mathrm{~V}$. The Xray energy resolution derived from this figure is approximately $20 \% \mathrm{FWHM}$. As expected, the 
argon escape peak appears at $5.0 \mathrm{keV}$. It is noted however that the pulse height spectrum shown in figure 9 contains a prominent low energy tail. This features arises when only partial events are recorded within the active area of the MSGC, part of the charge being lost at the MSGC boundaries by electron diffusion [13].

\subsection{Gain Stability}

Figure 10 shows the short term effective gain stability versus time after switching on the GEMMSGC system. Here the MSGC gain contribution was kept at zero $\left(\mathrm{V}_{\mathrm{a}}=+250 \mathrm{~V}\right)$ whilst the GEM mesh was operated at an initial effective gain of approximately 1000. The counter gas mixtures used for assessing the short term effective gain stability were $\operatorname{Ar}(52 \%)-\mathrm{DME}(48 \%)$ and $\operatorname{Ar}(75 \%)-\operatorname{IB}(25 \%)$. The short term effective gain variation is strongly dependent upon the GEM hole geometry [9] as well as ambient pressure $(\mathrm{P})$ and temperature $(\mathrm{T})$ [14]. In the present case, negligible $\mathrm{P} / \mathrm{T}$ excursions were encountered during the gain stability studies.

As shown in figure 10, data taken with $\operatorname{Ar}(52 \%)-\operatorname{DME}(48 \%)$ showed an increase in gain of approximately $9 \%$ from its initial value over a period of nearly 2 hours $\left(\Delta_{\mathrm{GEM}}=465 \mathrm{~V}, \mathrm{E}_{\mathrm{d}}=-\right.$ $\left.0.33 \mathrm{kV} / \mathrm{cm}, \mathrm{E}_{\mathrm{I}}=2.2 \mathrm{kV} / \mathrm{cm}\right)$. However, when using $\operatorname{Ar}(75 \%)-\mathrm{IB}(25 \%)$, gain increased by approximately $4 \%$ over the same period $\left(\Delta_{\mathrm{GEM}}=400 \mathrm{~V}, \mathrm{E}_{\mathrm{d}}=-0.33 \mathrm{kV} / \mathrm{cm}, \mathrm{E}_{\mathrm{C}}=2.8 \mathrm{kV} / \mathrm{cm}\right)$. The gain stabilisation time found here is much longer than other studies [9 and 15] where the corresponding values were only a few minutes. Furthermore, the exceptionally long settling time experienced during this study when using DME is similar to that found elsewhere [16].

Kapton based GEM devices are more permeable to moisture and gasses when compared to other materials such as liquid crystal polymers (LCP). One possible mechanism for gain enhancement with DME after switching on the GEM-MSGC system could be due to the absorption of DME into the kapton holes. This process could lower the tension of the GEM mesh thus decreasing the induction gap thereby increasing the induction field. Consequently, the effective gain would increase as was shown in figure 4. Preliminary measurements seem to confirm this hypothesis where kapton and LCP samples held in frames were exposed to DME for a number of days. It was found that the kapton sample increased in weight by $0.3 \%$ with evidence of physical softening as seen by drooping of the central region when supported at the edges. In contrast, the LCP samples remained robust without any changes in weight.

By combining the lower effective gain increase by using $\operatorname{Ar}(75 \%)-\mathrm{IB}(25 \%)$ and operating at a higher induction field $\left(\mathrm{E}_{\mathrm{I}}=3.7 \mathrm{kV} / \mathrm{cm}\right)$, no noticeable changes were observed after switching on and tracking the effective gain for up to nearly 2 hours.

\subsection{Conclusion}

A GEM-MSGC combination was successfully used to investigate parameters relevant to the Xray detection. These included measurements of the relative pulse height as a function of the drift field, the effective gain and X-ray energy resolution as a function of the drift field, the induction field, the voltage applied across the GEM holes and that applied at the MSGC anodes and cathodes.

It was confirmed that the relative pulse height was unaffected by the induction field and the region of good electron transparency was extended by applying a higher voltage across the GEM holes. Using the GEM-MSGC system the effective gains exceeded 100,000 whilst shifting the gain-voltage characteristics of the MSGC towards much lower voltages. However, 
good X-ray energy resolution was only possible up to an effective gain of 30,000 . At this limit, $18 \%$ FWHM X-ray energy resolution was achieved at $8.05 \mathrm{keV}$.

Obtaining $18 \% \mathrm{FWHM}$ at $8.05 \mathrm{keV}$ at an effective gain of 30,000 represents a considerable improvement over the previous studies using the MSGC alone [7 and 10] where the onset of $\mathrm{X}$-ray energy resolution degradation began at a gain of only a couple of thousand. In view of these findings, the present study is now being extended to investigate helium-isobutane mixtures used in synchrotron radiation based EXAFS experiments where gain tends to be a limiting factor particularly involving the sub-keV X-rays.

This study also showed that the DME is not a particularly good gas component for a kapton based GEM mesh. Applications demanding the use of a the DME counter gas mixture must therefore seek other GEM materials such as a suitable LCP. It was also demonstrated that by using $\operatorname{Ar}(75 \%)-\mathrm{IB}(25 \%)$ particularly at a higher induction field allowed the GEM-MSGC operation without any short term variations in the effective gain.

\section{Acknowledgement}

JAM acknowledges Drs. N.J. Rhodes, E.M. Schooneveld, G.E. Derbyshire, D.M. Duxbury and $\mathrm{R}$. Stephenson for their valuable discussions during the course of this study as well as support arising from CCLRC Rutherford Appleton Laboratory's ISIS, CFI and the DEG Group. JAM also wishes to acknowledge support received from Sheffield University's Dark Matter Group (Particle Astrophysics) through Prof. N. Spooner and Dr. T.B. Lawson.

\section{References}

[1] F. Sauli, “GEM: A new concept for electron amplification in gas detectors”, Nucl. Instrum. and Meth. A386 (1997) 531-534.

[2] F. Sauli, "Construction, test and commissioning of the triple-gem tracking detector for compass", Nucl. Instrum. and Meth.A490 (2002) 177-203.

[3] B.Yu, G. De Geronimo, V. Redeka, G.C. Smith, C. Thorn, "A Micro TPC for the LEGS Experiment", Presented at the IEEE Nuclear Science Symposium, 19th-25th October 2003, Portland, USA.

[4] R. Bellazzini et al., "Ultra soft x-ray two dimensional plasma imaging system based on gas electron multiplier detector with pixel readout", Rev. Sci. Instrum., (72) No2. (2001) 1372-1378.

[5] M. Klein and C.J. Schmidt, "CASCADE: Vision of a Modern Neutron Area Detector", Proceedings of the $5^{\text {th }}$ TECHNI meeting, $15^{\text {th }}$ May 2002, Bonn, Germany.

[6] T.L. Van Vuure, C.W.W. Van Eijk, F. Fraga et al., "High-Pressure GEM operation aiming at thermal neutron detection”, IEEE Trans. Nucl. Sci. 48 (4), Part 1 (2001) 1092-1094. 
[7] J.A. Mir et al., "Optimising the design of gas microstrip detectors for soft X-ray detection", Presented at the IEEE Nuclear Science Symposium, 4th-10th November 2001, San Diego, USA. See also IEEE Trans. Nucl. Sci. 49 (3), Part 2, (2002) 894-898.

[8] O. Bunemann, T.E. Cranshaw, and J.A. Harvey, Can. Jour. Res., 27 (1949) 191.

[9] F. Sauli et al., "Further developments and beam tests of the gas electron multiplier (GEM)", Nucl. Instrum. and Meth.A419(1998) 410-417.

[10] J.A. Mir et al., "High-gain microstrip counters for soft X-ray detection”, Presented at the Sixth International Conference on Position Sensitive Detectors, 9-13 September 2002, Leicester, UK. See also Nucl. Instrum. and Meth. A 513, (1-2), (2003) 273-276.

[11] A. Breskin, R. Chechik, "Detection of single electrons and low ionization radiation with low pressure multistep chambers", IEEE Trans. Nucl. Sci. NS-32 (1), (1984) 504-509.

[12] B.D. Ramsey, M.C. Weisskopf, "On the energy resolution obtained with a multistep proportional counter", Nucl. Instrum. and Meth.A248 (1986) 550-556.

[13] J.E Bateman, J.F. Connolly, G.E. Derbyshire, D.M. Duxbury, J.A. Mir, E.J. Spill, R. Stephenson,"Energy resolution in X-ray detecting micro-strip gas counters", Nucl. Instrum. and Meth. 484 (1-3), (2002) 384-395.

[14] J.A. Mir et al., "Studies of the gain properties of microstrip gas counters relevant to their application as X-ray and electron detectors", Presented at the IEEE Nuclear Science Symposium, 4th-10th November 2001, San Diego, USA. See also IEEE Trans. Nucl. Sci. 49 (4), Part 1, (2002) 1644-1650.

[15] F. Sauli et al.,"New observations with the gas electron multipliers (GEM)", Nucl. Instrum. and Meth. A396 (1997) 50-66.

[16] T.L. Van Vuure et al.,"Limits to proportionality in the double GEM detector", Nucl. Instrum. and Meth. A477 (2002) 37-40.

\section{Figure Captions}

1. Schematic diagram showing the GEM-MSGC arrangement used in the present study.

2. A plot of the relative pulse height and $\mathrm{X}$-ray energy resolution as a function of drift field using $\operatorname{Ar}(75 \%)-\operatorname{IB}(25 \%)\left(\Delta_{\mathrm{GEM}}=400 \mathrm{~V}, \mathrm{~V}_{\mathrm{a}}=+250 \mathrm{~V}\right.$ and $\left.\mathrm{E}_{\mathrm{G}}=2.5 \mathrm{kV} / \mathrm{cm}\right)$.

3. A plot of the relative pulse height as a function of drift field using $\operatorname{Ar}(75 \%)-\operatorname{IB}(25 \%)$ for a number of different $\Delta_{\mathrm{GEM}}$ voltages and induction fields, $\mathrm{E}$.

4. A plot of the effective gain and $\mathrm{X}$-ray energy resolution as a function of the induction field using $\operatorname{Ar}(75 \%)-\mathrm{IB}(25 \%)$ for $\Delta_{\mathrm{GEM}}=250 \mathrm{~V}$ and $350 \mathrm{~V}$. In both cases $\mathrm{V}_{\mathrm{c}}=-622 \mathrm{~V}$ and $\mathrm{E}_{\mathrm{d}}=-$ $0.25 \mathrm{kV} / \mathrm{cm}$.

5. A plot of the effective gain as a function of the anode cathode potential $\mathrm{V}_{\mathrm{a}}$, using $\operatorname{Ar}(75 \%)$ $\mathrm{IB}(25 \%)$ with $\Delta_{\mathrm{GEM}}=0 \mathrm{~V}, 250 \mathrm{~V}, 350 \mathrm{~V}$ and $500 \mathrm{~V}$. 
6. A plot of the X-ray energy resolution at $8.05 \mathrm{keV}$ as a function of the anode cathode potential $\mathrm{V}_{\mathrm{a}}$, using $\operatorname{Ar}(75 \%)-\mathrm{IB}(25 \%)$ with $\Delta_{\mathrm{GEM}}=0 \mathrm{~V}, 250 \mathrm{~V}, 350 \mathrm{~V}$ and $500 \mathrm{~V}$.

7. A plot of the effective gain as a function of the voltage across the GEM holes $\left(\Delta_{\mathrm{GEM}}\right)$ using $\operatorname{Ar}(75 \%)-\mathrm{IB}(25 \%)$ for $\mathrm{V}_{\mathrm{a}}=+250 \mathrm{~V},+465 \mathrm{~V}$ and $+622 \mathrm{~V}$.

8. A plot of the X-ray energy resolution at $8.05 \mathrm{keV}$ as a function of the voltage across the GEM holes $\left(\Delta_{\mathrm{GEM}}\right)$ using $\operatorname{Ar}(75 \%)-\mathrm{IB}(25 \%)$ for $\mathrm{V}_{\mathrm{a}}=+250 \mathrm{~V},+465 \mathrm{~V}$ and $+622 \mathrm{~V}$.

9. A typical pulse height spectrum for $8.05 \mathrm{keV} \mathrm{X-rays} \mathrm{using} \mathrm{GEM} \mathrm{amplification} \mathrm{alone} \mathrm{in}$ $\operatorname{Ar}(75 \%)-\mathrm{IB}(25 \%)$. In this case, $\Delta_{\mathrm{GEM}}=400 \mathrm{~V}, \mathrm{~V}_{\mathrm{a}}=+250 \mathrm{~V}, \mathrm{E}_{\mathrm{d}}=-0.33 \mathrm{kV} / \mathrm{cm}$ and $\mathrm{E}_{\mathrm{I}}=2.5 \mathrm{kV} / \mathrm{cm}$.

10. A typical short term settling behaviour of a GEM operated at approximately 1000 showing the variation of the effective gain using $\operatorname{Ar}(52 \%)-\mathrm{DME}(48 \%)$ and $\operatorname{Ar}(75 \%)-\operatorname{IB}(25 \%)$ at a number of different induction fields. 


\section{Figure 1}

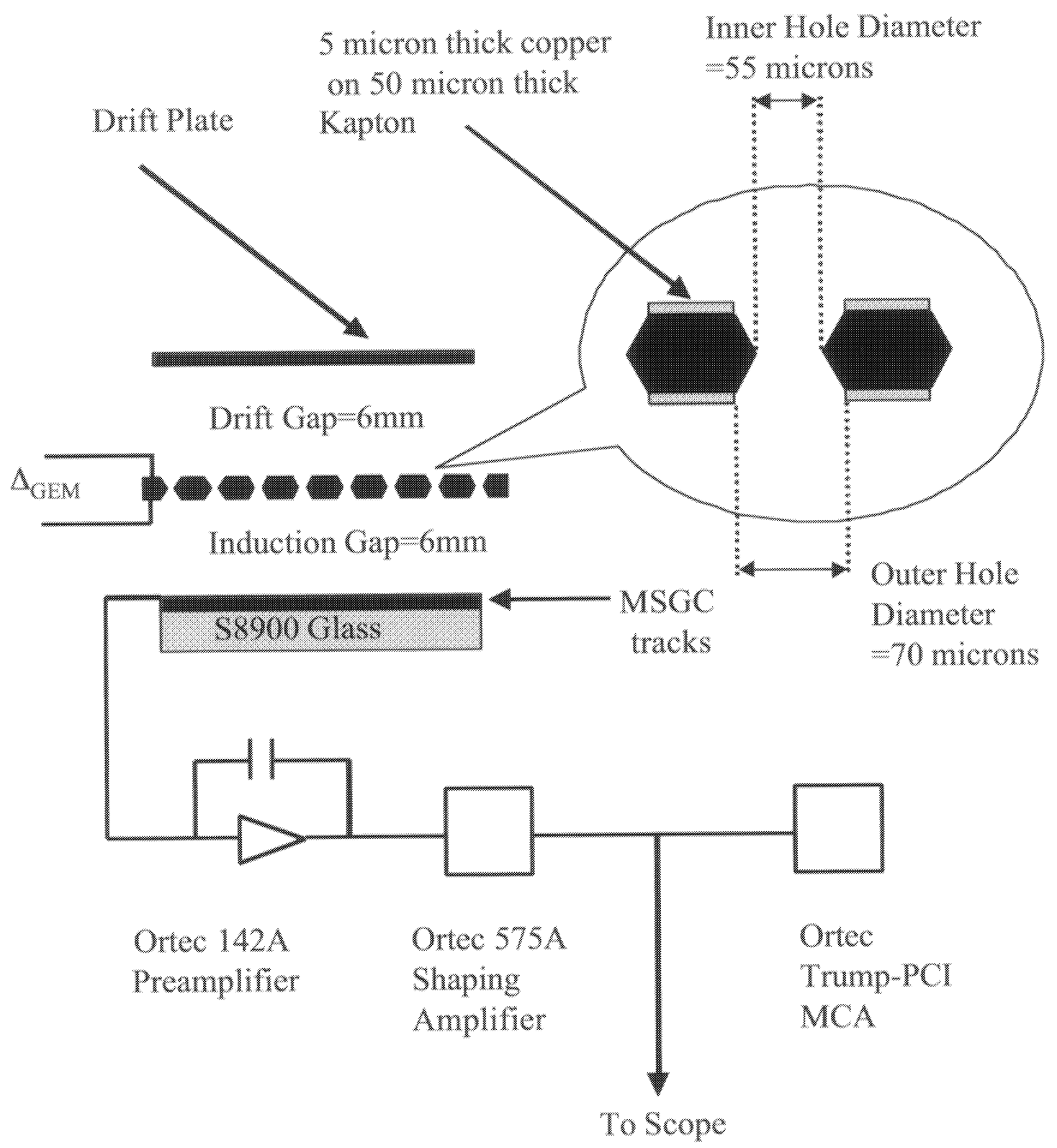


Figure 2

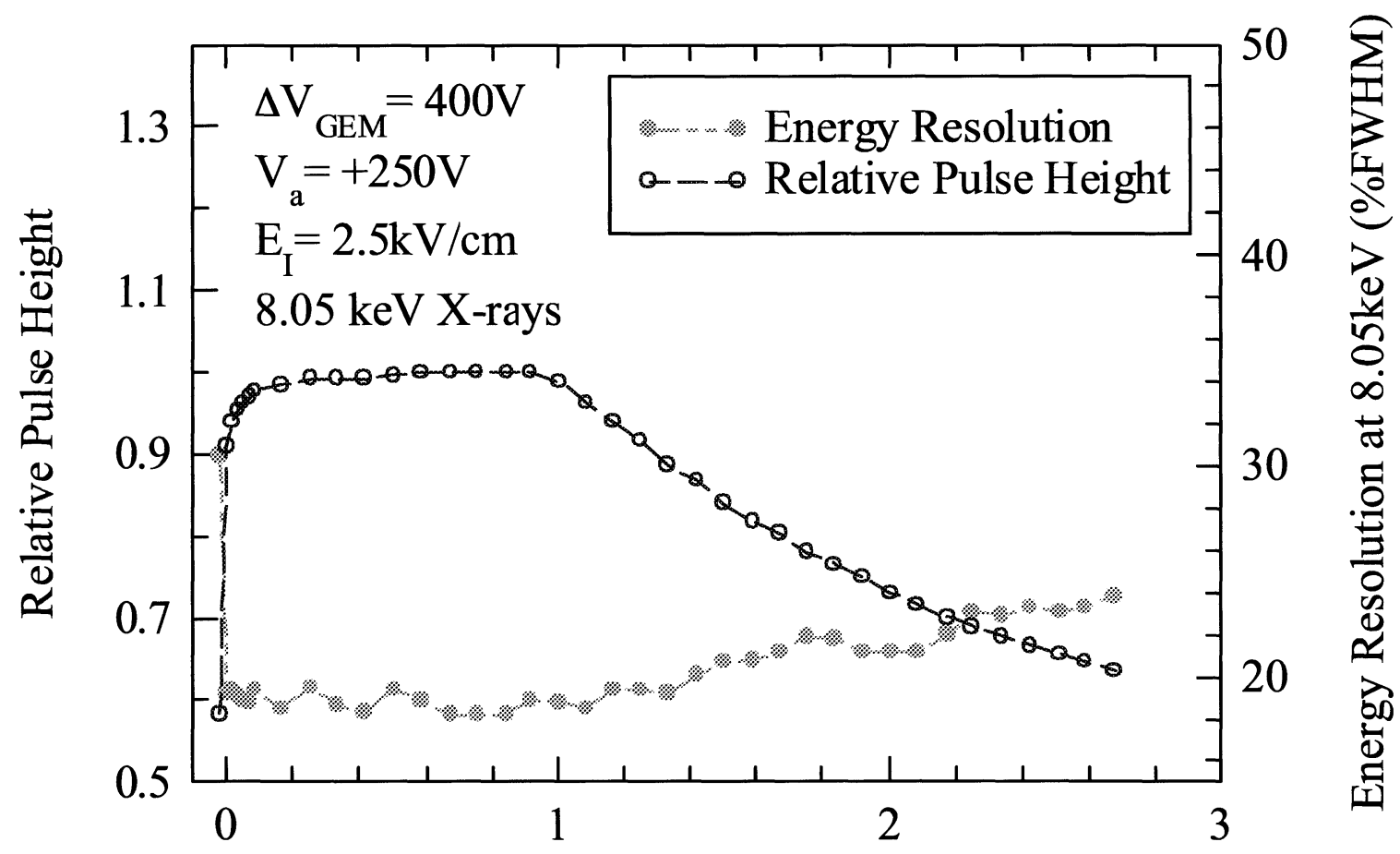

Drift Field $-E_{d}(k V / c m)$

Figure 3

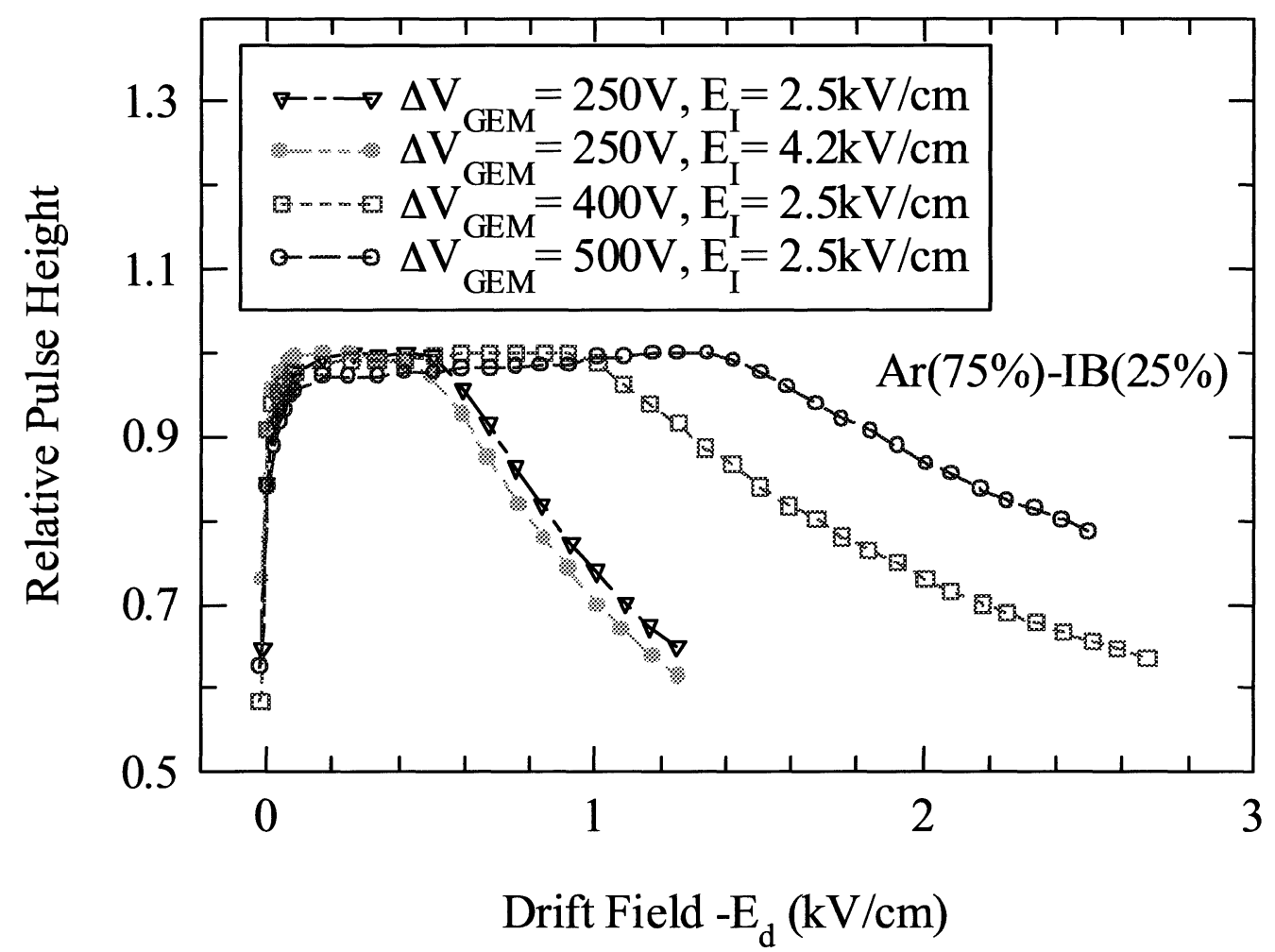


Figure 4

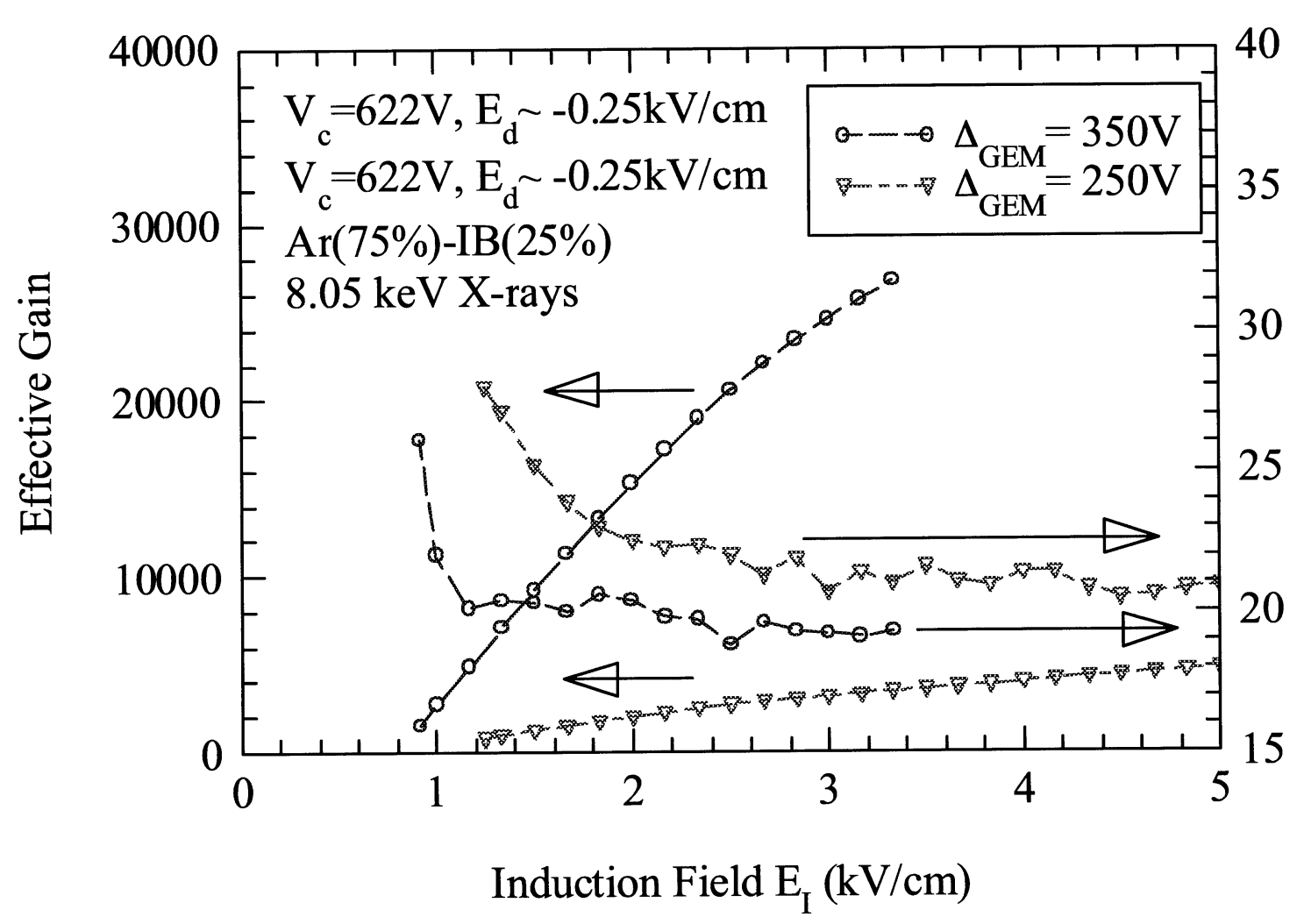

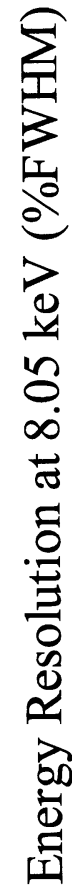

Figure 5

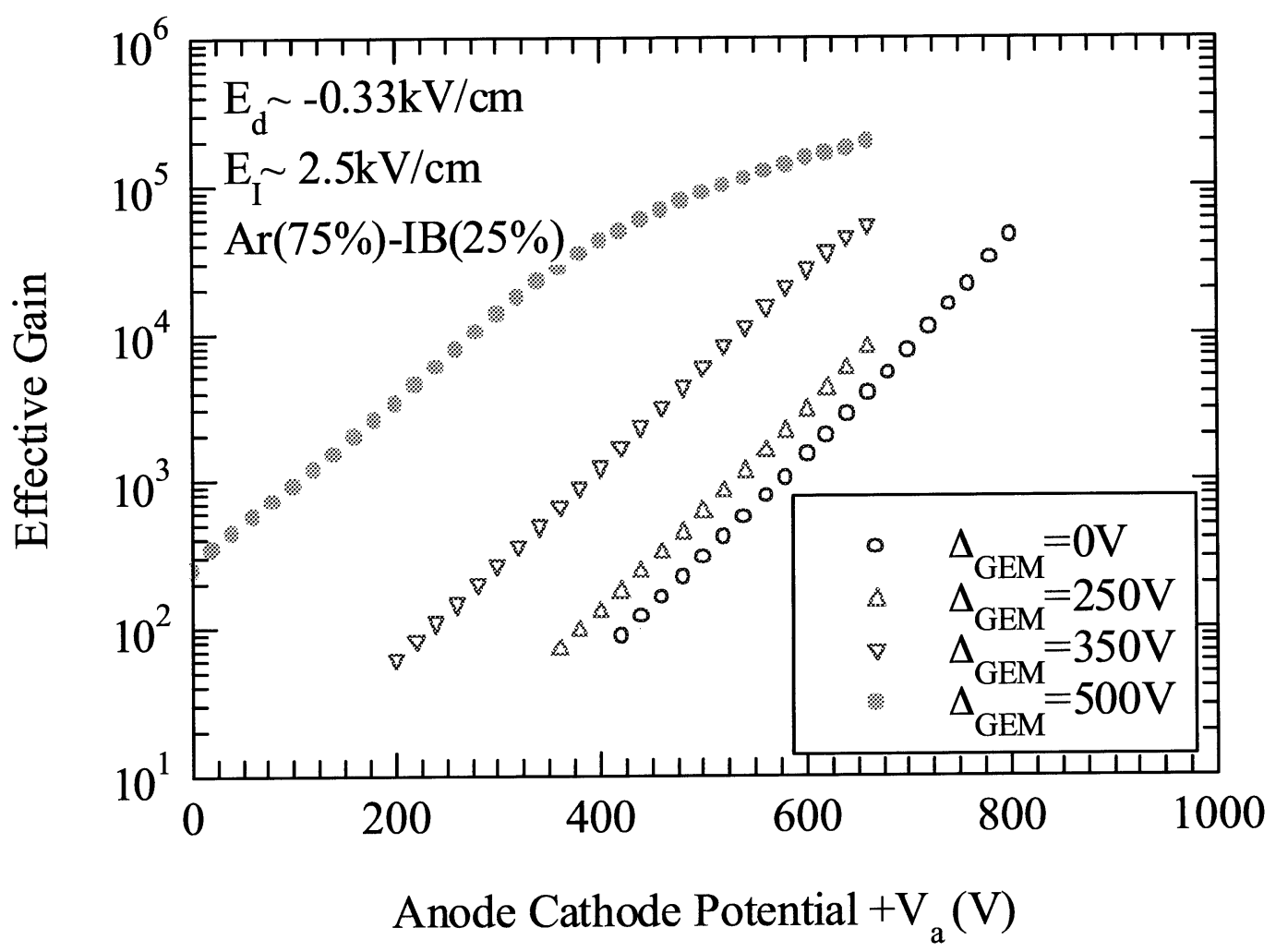


Figure 6

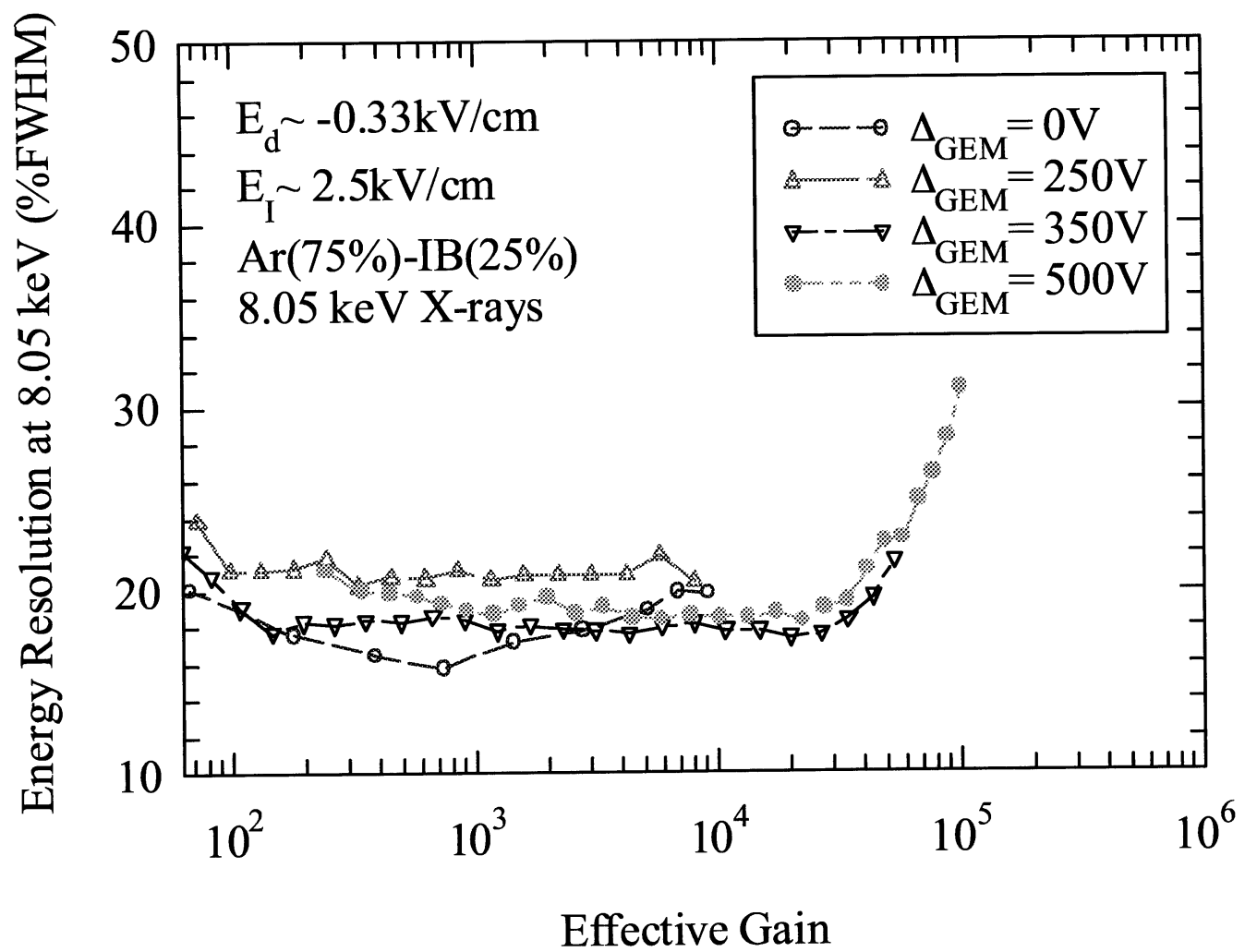

Figure 7

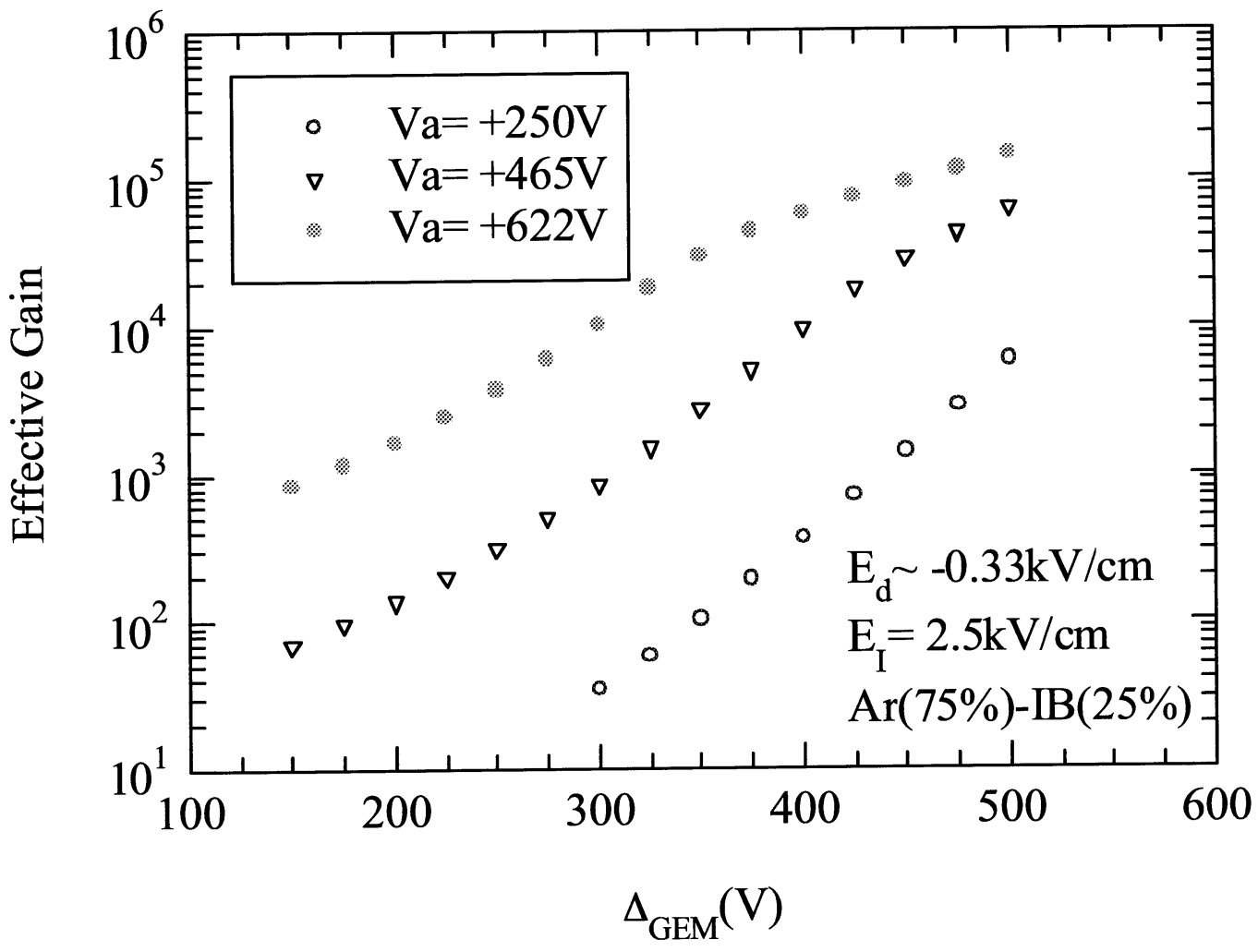


Figure 8

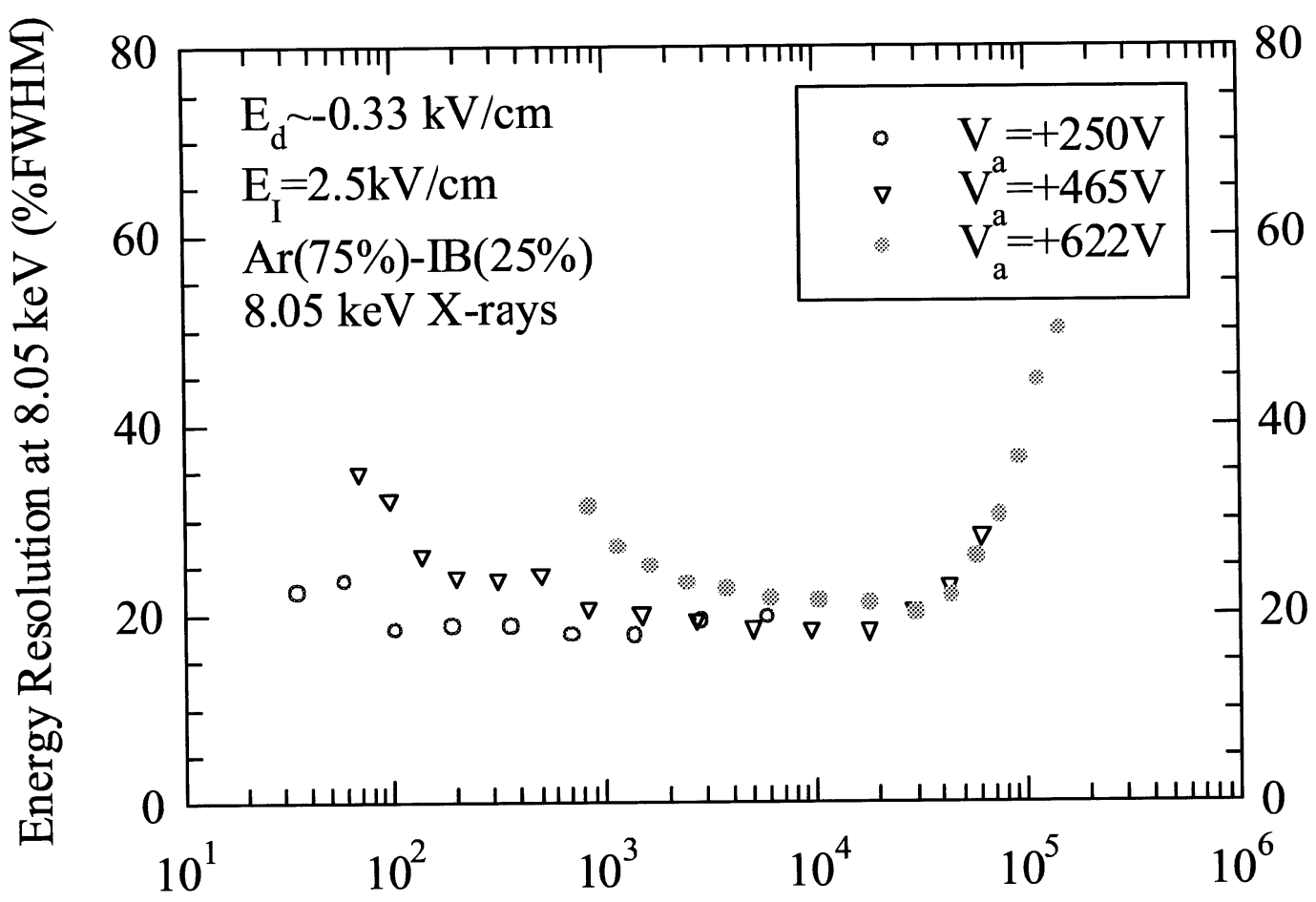

Effective Gain

Figure 9

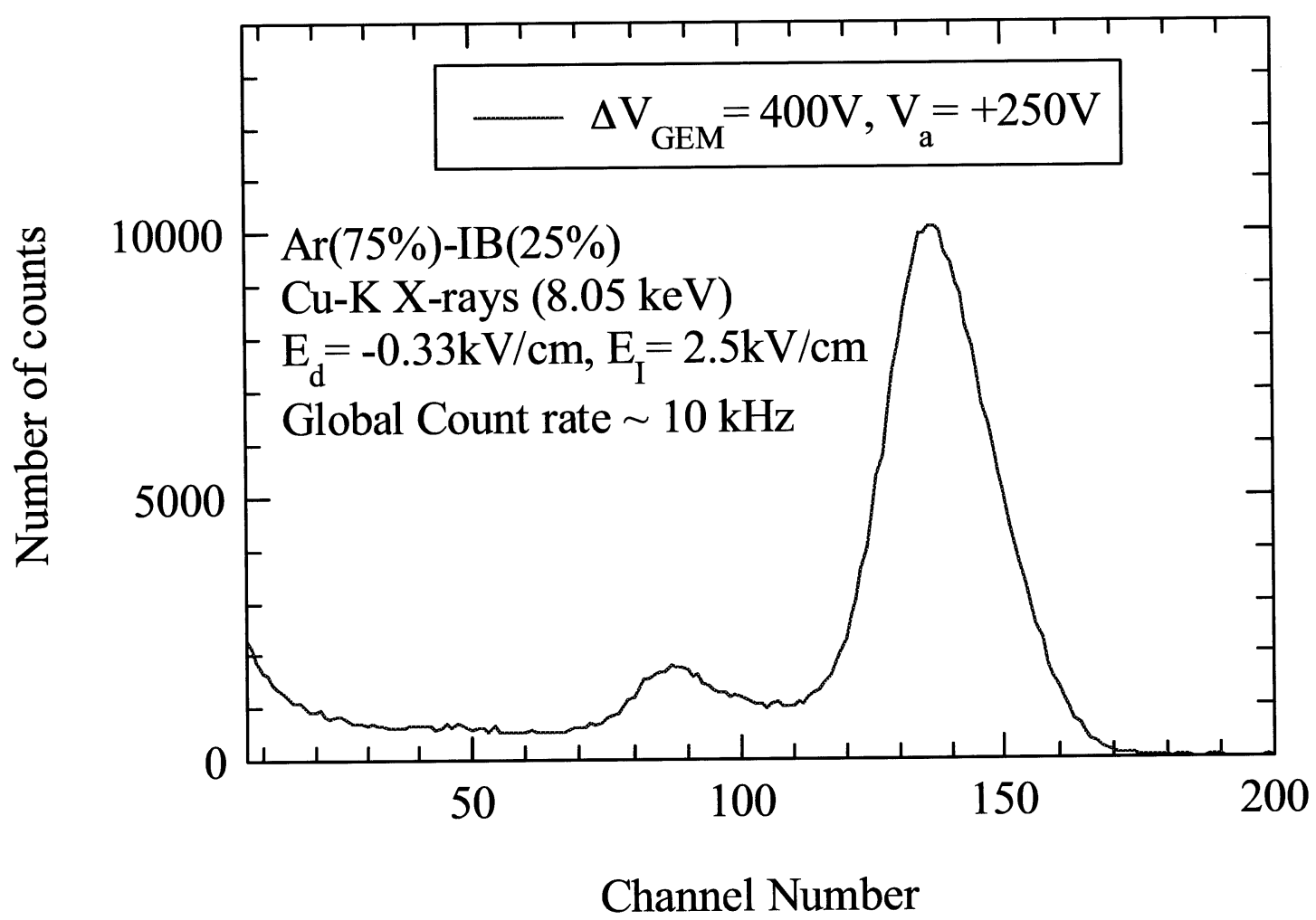


FIGURE 10

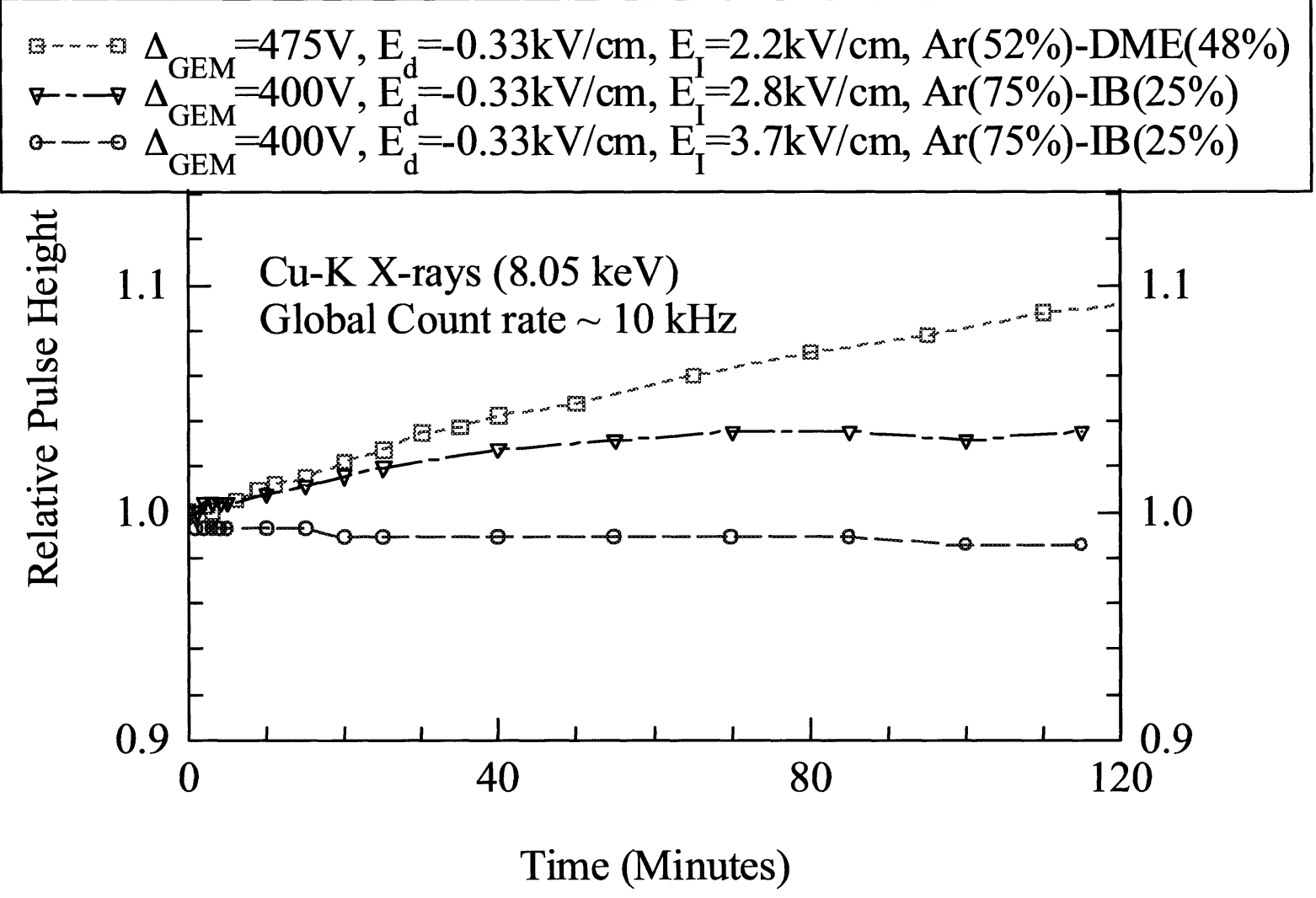


